\title{
Alpha-cluster model of atomic nuclei
}

\author{
Zbigniew Sosin ${ }^{1 \dagger}$, Jan Błocki ${ }^{2}$, Jinesh Kallunkathariyil ${ }^{1, a}$, Jerzy Lukasik ${ }^{3}$, and Piotr Pawłowski ${ }^{3}$ \\ 1 M. Smoluchowski Institute of Physics, Jagiellonian University, Łojasiewicza 11, 30-348 Kraków, Poland \\ 2 NCBJ, Theoretical Physics Division (BP2), Świerk, Poland \\ 3 IFJ PAN, Kraków, Poland
}

Received: 17 August 2015 / Revised: 24 February 2016

Published online: 5 May 2016

(C) The Author(s) 2016. This article is published with open access at Springerlink.com

Communicated by A. Ramos

\begin{abstract}
The description of a nuclear system in its ground state and at low excitations based on the equation of state (EoS) around normal density is presented. In the expansion of the EoS around the saturation point, additional spin polarization terms are taken into account. These terms, together with the standard symmetry term, are responsible for the appearance of the $\alpha$-like clusters in the ground-state configurations of the $N=Z$ even-even nuclei. At the nuclear surface these clusters can be identified as alpha particles. A correction for the surface effects is introduced for atomic nuclei. Taking into account an additional interaction between clusters the binding energies and sizes of the considered nuclei are very accurately described. The limits of the EoS parameters are established from the properties of the $\alpha,{ }^{3} \mathrm{He}$ and $t$ particles.
\end{abstract}

\section{Introduction}

In 1936 Bethe and Bacher [1] and in 1938 Hafstad and Teller [2] predicted that alpha-particle structures $[3,4]$ could be present in atomic nuclei. Convincing arguments for the existence of such structures were provided by systematics of the binding energies of the even-even nuclei with equal number of protons and neutrons (see also [5]), as well as by systematics of the binding energy of the additional neutron in nuclei like ${ }^{9} \mathrm{~B},{ }^{13} \mathrm{C}$ and ${ }^{17} \mathrm{O}$. The latter systematics could be explained by assuming that the valence neutron moves in a multi-center potential with centers identified with alpha particles. Today, from a commonly accepted point of view one considers that alphaparticle clusters can appear in the nuclear matter at subsaturation densities. Such conditions are met, e.g., at the nuclear surface where, in not too heavy nuclei, almost the whole nuclear mass is concentrated.

Subtle changes of the ground-state energy of atomic nuclei as a function of the nucleon number can be described by the shell model, which neglects correlations between nucleons. The main idea of the present approach is to verify whether these subtle changes, in the case of $n$-alpha nuclei, could be attributed to the appearance of alpha-like clusters which modify the structure of the wave function and introduce strong correlations between nucle-

\footnotetext{
${ }^{a}$ e-mail: jinesh.kallunkathariyil@gmail.com

$\dagger$ Deceased.
}

ons. In such an approach the system in its ground state behaves like a crystal, with stationary configuration and shape and with definite distances between the wave function centers for a given nucleus. The subtle changes of the binding energy due to appearance of alpha-like clusters in the wave functions of $n$-alpha nuclei will be determined within an extended version of the model proposed in [6].

It will be shown in the following that the hypothesis of alpha structures in the $n$-alpha nuclei can indeed describe the binding energy systematics of Bethe $[1,2]$. It will be also shown that the hypothesis of the alpha structures helps to understand the evolution of the root-mean-square (rms) radii and of the density profiles as a function of the number of alpha particles in the $n$-alpha nuclei.

The paper is organized as follows: sect. 2 describes the adopted interactions in the nuclear matter. Section 3 presents constraints on the introduced parametrization resulting from the properties of light charged particles. Section 4 is devoted to the ground-state properties of $n$-alpha nuclei. First, it investigates the effects of clusterization on the ground-state configurations. Then, some corrections to the Hamiltonian, related to the many-body character of the nuclear interaction and to the bosonic nature of the wave function, are introduced. It will be shown that the introduced corrections make the structural corrections stronger. Finally, the model predictions for binding energies, rms radii and density profiles are presented and compared with the experimental values. Section 5 presents the conclusions. 


\section{Interactions in nuclear matter around saturation}

In order to predict correctly a state and evolution of a nuclear system, a reliable description of the interaction between nucleons is needed. In the case of a strong interaction it is not an easy task. As we know, the fundamental theory governing the nucleon-nucleon interactions is the quantum chromodynamics (QCD), where interactions are calculated from the physics of quarks and gluons. Unfortunately, very limited progress has been achieved in the application of QCD for the description of the interaction between quark-gluon clusters. Therefore, a phenomenological approach, associated to some specific conditions has been applied. Since we are interested in the low-energy region in which nucleons are not excited internally, one can treat nucleons as quasi-elementary particles. Additionally, if we apply a non-relativistic approximation, the mutual nucleon interaction can be described by a respective potential. As can be found in the text-books, details of the form of such a potential are deduced from nuclear interaction symmetries and the mechanism of exchange of bosons.

For a final test of this type of potential and the determination of its parameters, one uses a comparison with the experimental data. Here the nucleon-nucleon scattering data or light-nuclei properties are usually used. A general conclusion from this kind of research is that a simple two-body potential is not sufficient to describe the behavior of the system of more than two nucleons. One should add at least the three-body force. In particular, in the nuclear system, the three-body force is acceptable because the strong interaction is generated by the exchange process of gluons. The $n$-body interaction can be interpreted as coming from the multi-pion exchange processes.

These considerations show the difficulties in the description of even the simplest nuclear system. The extension of the description of the interaction by the three-body forces shows that the exchanged particles (bosons) play a significant role in the interaction process.

In order to describe the dynamics of the system, one has to express the change of energy associated with its evolution. In the description of energy through the potential one has to define its form. Hence the wave function of a system of identical particles must be either symmetric or antisymmetric and it must be taken into account when defining the energy of the ground state. In the case of the fermionic systems one should use an antisymmetric wave function which is a serious difficulty in the description of the collision dynamics. Some proposals to solve this problem are shown for instance in [7-9]. Other approaches, introducing some kinds of momentum-dependent Pauli potentials and Pauli-blocking schemes, which are meant to mimic the effects of antisymmetrization and are much less time consuming, are also proposed, see, e.g., [10-14].

Therefore, in the present approach, we propose a description in which particles are distinguishable and the energy associated with the fermionic motion affects the resulting energy density which is parametrized and used instead of the potential. Such a concept is present in the description of the liquid drop model (LDM) and in some density functional models, e.g., in [15]. It is obvious that such an approach requires the knowledge of the local density of matter and the knowledge of the wave function describing the system. In the present description we use a standard one, commonly applied in such cases, i.e. the wave function is defined as a simple product of minimal wave packets (eq. (1)), as, e.g., in [16]. The main argument for disregarding the antisymmetrization in the present approach is the form of the proposed EoS which includes the spin and isospin terms. These terms force the unlike nucleons to form "bosonic" alpha-like clusters for which, presumably, the antisymmetrization may no longer play a decisive role.

Each minimal wave packet in the adopted wave function has a defined spin and a specific charge for a selected quantization axis:

$$
\begin{aligned}
\Phi & =\prod_{k=1}^{A}{ }^{k} \phi_{I_{k} S_{k}}, \\
{ }^{k} \phi_{I_{k} S_{k}} & =\frac{1}{\left(2 \pi \sigma_{k}^{2}(r)\right)^{3 / 4}} \exp \left(\frac{-\left(\mathbf{r}_{k}-\left\langle\mathbf{r}_{k}\right\rangle\right)^{2}}{4 \sigma_{k}^{2}(r)}+\frac{i}{\hbar} \mathbf{r}_{k}\left\langle\mathbf{p}_{k}\right\rangle\right),
\end{aligned}
$$

where $\sigma_{k}^{2}(r),\left\langle\mathbf{r}_{k}\right\rangle,\left\langle\mathbf{p}_{k}\right\rangle$ are the width (the position variance of the $k$-th nucleon) of a Gaussian wave packet and the mean position and mean momentum of each of the $A$ nucleons, respectively. Every partial wave function is labeled by $I_{k}=n$ or $I_{k}=p$ and $S_{k}=\uparrow$ or $S_{k}=\downarrow$, denoting the isospin and spin (or precisely, their projections on a specified quantization axis) associated with a given nucleon. Variables $\left\langle\mathbf{r}_{k}\right\rangle,\left\langle\mathbf{p}_{k}\right\rangle$ and $\sigma_{k}^{2}(r)$ are, in general, time-dependent parameters describing the wave functions.

In the present approach the nuclear matter is treated as a four-component fluid characterized by the respective densities:

$-\rho_{p \uparrow}$ for protons with spin up;
$-\rho_{p \downarrow}$ for protons with spin down;
$-\rho_{n \uparrow}$ for neutrons with spin up;
$-\rho_{n \downarrow}$ for neutrons with spin down.

Density distributions in the system are uniquely determined by parameters of the wave function (mean position, mean momentum and variance of the position of each nucleon). The description of the system evolution due to the time evolution of these parameters is, in general, defined by the Dirac-Frenkel time-dependent variational principle [17]. This approach describes the interaction in a selfconsistent way.

Assuming that nucleons are moving inside the nuclear system, the average energy of the system is a sum of the average potential energy and of the kinetic energy associated with the fermionic motion. This is the case in which one disregards the energy of the ordered motion, which, for the low excitations of the system, is considered later on as a correction (see formula (14)).

According to the above idea we now present a method for determining the average energy associated with each nucleon. For the description of this average energy we assume the existence of a scalar field $\varepsilon\left(\rho_{p \uparrow}, \rho_{p \downarrow}, \rho_{n \uparrow}, \rho_{n \downarrow}\right)$, 
determined by the local densities $\rho_{p \uparrow}(\mathbf{r}), \rho_{p \downarrow}(\mathbf{r}), \rho_{n \uparrow}(\mathbf{r})$, $\rho_{n \downarrow}(\mathbf{r})$.

If, for every $k$-th nucleon, the corresponding wave packet ${ }^{k} \phi_{I_{k} S_{k}}$ determines the probability $P_{k}(\mathbf{r})=$ $\left|{ }^{k} \phi_{I_{k} S_{k}}\right|^{2}$ of finding a nucleon at a given point $\mathbf{r}$, then it is assumed that the average energy $e_{k}$ associated with this nucleon is defined by the mean value and variance of the field $\varepsilon\left(\rho_{p \uparrow}, \rho_{p \downarrow}, \rho_{n \uparrow}, \rho_{n \downarrow}\right)$ and can be expressed as

$$
e_{k}=\langle\varepsilon\rangle_{k}+\lambda \sigma_{k}(\varepsilon)
$$

where

$$
\begin{aligned}
\langle\varepsilon\rangle_{k} & =\int P_{k}(\mathbf{r}) \varepsilon\left(\rho_{p \uparrow}, \rho_{p \downarrow}, \rho_{n \uparrow}, \rho_{n \downarrow}\right) \mathrm{d}^{3} \mathbf{r} \\
\sigma_{k}^{2}(\varepsilon) & =\int P_{k}(\mathbf{r})\left(\varepsilon\left(\rho_{p \uparrow}, \rho_{p \downarrow}, \rho_{n \uparrow}, \rho_{n \downarrow}\right)-\langle\varepsilon\rangle_{k}\right)^{2} \mathrm{~d}^{3} \mathbf{r}
\end{aligned}
$$

and $\lambda$ is a parameter related to the surface energy, similarly as in [18].

The EoS of the nuclear matter is defined for an infinite system, assuming its homogeneity and isotropy. For such a matter, the variance of the associated field $\varepsilon\left(\rho_{p \uparrow}, \rho_{p \downarrow}, \rho_{n \uparrow}, \rho_{n \downarrow}\right)$ vanishes and the average value of the energy per nucleon is determined by the mean value of the field alone. The field $\varepsilon\left(\rho_{p \uparrow}, \rho_{p \downarrow}, \rho_{n \uparrow}, \rho_{n \downarrow}\right)$ is in this case equivalent to the EoS of the nuclear matter. Thus, the functional form of the field $\varepsilon\left(\rho_{p \uparrow}, \rho_{p \downarrow}, \rho_{n \uparrow}, \rho_{n \downarrow}\right)$ can be obtained from the EoS by applying the local density approximation.

In the present work we use a 12-parameter cubic form of the EoS proposed in [6]. In the vicinity of the saturation density it can be approximated using a second-order Taylor expansion:

$$
\begin{aligned}
e= & e_{00}+\frac{K_{0}}{18} \xi^{2} \\
& +\delta^{2}\left(e_{I 0}+\frac{L_{I}}{3} \xi+\frac{K_{I}}{18} \xi^{2}\right) \\
& +\left(\eta_{n}^{2}+\eta_{p}^{2}\right)\left(e_{i i 0}+\frac{L_{i i}}{3} \xi+\frac{K_{i i}}{18} \xi^{2}\right) \\
& +2 \eta_{n} \eta_{p}\left(e_{i j 0}+\frac{L_{i j}}{3} \xi+\frac{K_{i j}}{18} \xi^{2}\right),
\end{aligned}
$$

where

$$
\begin{gathered}
\xi=\frac{\rho-\rho_{0}}{\rho_{0}}, \\
\delta=\frac{\rho_{n}-\rho_{p}}{\rho}, \\
\eta_{n}=\frac{\rho_{n \uparrow}-\rho_{n \downarrow}}{\rho}, \\
\eta_{p}=\frac{\rho_{p \uparrow}-\rho_{p \downarrow}}{\rho}
\end{gathered}
$$

in which $\rho$ and $\rho_{0}$ are the total nuclear matter density and the density of isospin and spin-balanced matter at saturation, respectively.
The first two terms in (6) are just a standard, 6-parameter form of the EoS approximation commonly used in the present-day experimental and theoretical studies, see, e.g., [19]. The first term describes the symmetric matter in a balanced system (zero isospin and spin), with $K_{0}$ being the compressibility of the symmetric nuclear matter. The second one is a standard approximation of the symmetry energy:

$$
e_{I}=e_{I 0}+\frac{L_{I}}{3} \xi+\frac{K_{I}}{18} \xi^{2}
$$

with $e_{I 0}$ being the Wigner constant and the coefficients $L_{I}$ and $K_{I}$ being the slope and curvature, respectively.

The last two terms of (6) are the main novelty of the present approach (see also [6]). In analogy to the isospin symmetry energy, they describe the spin symmetry energies for neutrons and protons separately:

$$
e_{i i}=e_{i i 0}+\frac{L_{i i}}{3} \xi+\frac{K_{i i}}{18} \xi^{2}
$$

and the energy of the mutual spin interaction of protons and neutrons:

$$
e_{i j}=e_{i j 0}+\frac{L_{i j}}{3} \xi+\frac{K_{i j}}{18} \xi^{2} .
$$

The terms with indices $i i$, i.e. the $e_{i i 0}, L_{i i}$ and $K_{i i}$ (constant, slope and curvature), describe the energy of protons or neutrons. Indices $i j$ indicate that the symmetry energy refers to the mutual interaction of protons and neutrons. The determination of the expansion parameters in (6) will be discussed in the next sections.

Using the field $\varepsilon\left(\rho_{p \uparrow}, \rho_{p \downarrow}, \rho_{n \uparrow}, \rho_{n \downarrow}\right)$ one can express the average value of the Hamiltonian for a given system of $A$ nucleons as

$$
\begin{aligned}
\langle\Phi|H| \Phi\rangle= & \sum_{k=1}^{k=A} \frac{\left\langle\mathbf{p}_{k}\right\rangle^{2}}{2 m_{N}}+\sum_{k=1}^{k=A}\langle\varepsilon\rangle_{k} \\
& +\lambda \sum_{k=1}^{k=A} \sigma_{k}(\varepsilon)+\left\langle\Phi\left|V_{C}\right| \Phi\right\rangle,
\end{aligned}
$$

where $m_{N}$ is the nucleon mass and the last term describes the Coulomb energy. As later on we will be searching for the ground-state configurations of the even-even nuclei with $N=Z$, it can be assumed that the average momenta of the wave packets $\left\langle\mathbf{p}_{k}\right\rangle$ are equal to zero and, thus, the first term in the expression (14) can be neglected. Following a standard approach (see, e.g., [19]) it is assumed that the parametrization of the EoS includes implicitly the kinetic contribution coming from the Fermi motion. As has been demonstrated in [18], the adopted cubic approximation of the EoS is flexible enough to reproduce typical predictions of sophisticated models of the nuclear matter which explicitly take into account the kinetic contribution resulting from the Fermi motion.

Since the local density can be written as

$$
\rho(\mathbf{r})=\sum_{k=1}^{k=A} P_{k}(\mathbf{r}),
$$


the average value of the Hamiltonian can be expressed as:

$$
\begin{aligned}
\langle\Phi|H| \Phi\rangle= & \int \varepsilon\left(\rho_{p \uparrow}, \rho_{p \downarrow}, \rho_{n \uparrow}, \rho_{n \downarrow}\right) \rho(\mathbf{r}) \mathrm{d}^{3} \mathbf{r} \\
& +\lambda \sum_{k=1}^{k=A} \sigma_{k}(\varepsilon)+\left\langle\Phi\left|V_{C}\right| \Phi\right\rangle .
\end{aligned}
$$

As one can see the Hamiltonian is a sum of three components, which can be interpreted as volume, surface and Coulomb energies. Therefore, the present description can be interpreted as a microscopic realization of the LDM.

Finding the ground-state configuration of a group of $A$ nucleons within the adopted parametrization of the wave function and for given parameters of the EoS, consists in finding the values of parameters $\sigma_{k}^{2}(r),\left\langle\mathbf{r}_{k}\right\rangle$, and $\left\langle\mathbf{p}_{k}\right\rangle$, which minimize the value of the Hamiltonian. The procedure of finding the ground-state configurations has been described in sect. 4 of [18]. We would like to emphasize that the proposed parametrization of the EoS, with two more terms in (6), is responsible for driving the nucleons of different types, $p \uparrow, p \downarrow, n \uparrow, n \downarrow$, to form quadruplets, i.e. to group into $\alpha$-like clusters during the minimization procedure. These two terms play a similar role as the standard symmetry term which attains a minimum when the neutron-proton asymmetry $\delta$ of (7) tends to zero, i.e. when the neutron-proton couples group together. This $\alpha$-like clustering property of (6) is shown in fig. 1.

The histogram in fig. 1 presents the evolution of the binding energy of 12 nucleons during the process of searching for the ground-state configuration which minimizes the model Hamiltonian (16). The 6 panels of the inset show "snapshots" of the projections of the mean positions of the nucleon wave packets onto the $x-y$ plane, at selected stages of the minimization procedure. Panel 1 presents the initial random configuration, panels $2-5$ some intermediate ones and panel 6 shows the final ground-state configuration of ${ }^{12} \mathrm{C}$. As can be seen, the minimum of the Hamiltonian is achieved for a configuration with three $\alpha$-like clusters. These clusters appear in the corners of an equilateral triangle. Note that in the figure the triangle is not perfectly equilateral due to the 2-dimensional projection.

It has to be emphasized, that if clusters are allowed to appear in the considered system the description has to be modified. Modifications of the Hamiltonian due to clustering will be discussed in sect. 4.2.

\section{Constraints on the EoS from the properties of light charged particles}

Ground-state properties of light charged particles, LCP, such as $d, t,{ }^{3} \mathrm{He}$ or $\alpha$, can provide important constraints on parameters which describe interactions in the nuclear matter for at least four reasons. First, one can assume that in their structure clusters of matter (obviously beyond nucleons) can be formed. Therefore, one may expect that clustering of matter has no effect on their binding energies and sizes. Second, these LCPs do not contain more

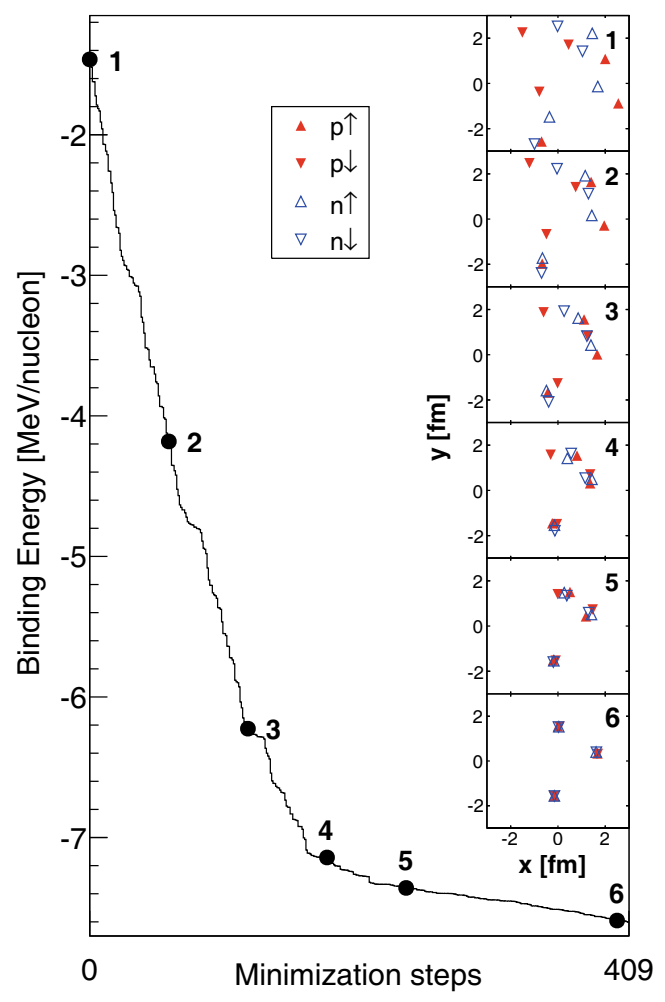

Fig. 1. Evolution of the binding energy of 12 nucleons $(3 \times p \uparrow$, $3 \times p \downarrow, 3 \times n \uparrow$ and $3 \times n \downarrow)$ during the minimization procedure. The insets 1-6 show the projections of the configurations of nucleons on the $x-y$ plane at selected stages of the minimization. Panel 1 represents the random initial configuration. Panel 6 shows the projection of the final ground-state configuration of ${ }^{12} \mathrm{C}$.

than one $\alpha$-particle and, thus, one can neglect the $\alpha$ - $\alpha$ interactions (see sect. 4.2) and use the simpler liquid-droplike Hamiltonian (16). Third, the measured density profiles of these light particles are relatively well described by Gaussian distributions, which is in line with the adopted parametrization of the wave function. Fourth, the simplicity of these particles assures the vanishing of some types of interactions in (6) and the selective increase of sensitivity to some other ones. For instance, parameters related to the isospin symmetry energy (second term in (6)) in principle do not affect the binding energy of a deuteron and alpha particle. Similarly, due to the saturation of the proton (neutron) spin interactions in ${ }^{3} \mathrm{He}(t)$, the mutual spin interaction (fourth term in (6)) vanishes in their case.

In the case of LCPs we can assume that the average value of the Hamiltonian is completely determined by the field $\varepsilon\left(\rho_{p \uparrow}, \rho_{p \downarrow}, \rho_{n \uparrow}, \rho_{n \downarrow}\right)$, by the Coulomb interaction and by the surface energy. This assumption may not be valid for more complex systems in which mutually interacting clusters can be formed, e.g., for ${ }^{8} \mathrm{Be} \rightarrow \alpha-\alpha$ or ${ }^{6} \mathrm{Li} \rightarrow \alpha-d$. For such systems one can expect additional interactions between clusters due to the specific many-body forces. The mean value of the Hamiltonian (16) depends on 13 parameters: 12 parameters of the field $\varepsilon(\mathbf{r})$ plus the surface tension parameter $\lambda$. Binding energies and rms radii of the ground states for $d, t,{ }^{3} \mathrm{He}$ and $\alpha$ provide 8 equations, thus 
an insufficient number of constraints for a unique choice of parameters describing the interaction. In the present section we will try to deduce a subspace of this 13-parameter space for which the above-mentioned properties of LCPs are reproduced.

\subsection{Constraints on $\rho_{0}, \mathrm{e}_{00}, \mathrm{~K}_{0}$ and $\lambda$ from the properties of an alpha particle}

It is clear that the greatest reduction of the number of parameters can be expected in the case of an alpha particle. Here, the spin- and isospin-dependent interactions are practically absent. Thus, properties of alpha particles should be described by four parameters only: $\rho_{0}, e_{00}, K_{0}$ and $\lambda$. Constraints on these parameters are imposed by the rms radius and the binding energy of an alpha particle. These parameters cannot be determined unambiguously. However, there is a flexibility in determining a twodimensional hypersurface, in the space $\rho_{0}, e_{00}, K_{0}$ and $\lambda$, in which the searched values of these parameters exist.

In order to determine this hypersurface the following $\chi^{2}$ variable is defined:

$$
\chi^{2}=\left(\frac{B_{\alpha e}-B_{\alpha m}}{B_{\alpha e}}\right)^{2}+\left(\frac{r_{\alpha e}-r_{\alpha m}}{r_{\alpha e}}\right)^{2},
$$

where $B_{\alpha e}, B_{\alpha m}, r_{\alpha e}, r_{\alpha m}$ are the experimental and model values of the binding energy and rms radius, respectively. Note that a variable defined in this way should be zero in the searched subspace.

In order to determine the correlations among the parameters in a searched subspace, the variables $\rho_{0}$ and $K_{0}$ are predefined on a grid, and the remaining two parameters are found by requiring that $\chi^{2}<10^{-7}$ for the model ground-state configurations of an alpha particle. The results are shown in fig. 2 .

The figure presents the constant saturation density curves as a function of the other three parameters specified on the axis, which ensure a correct binding energy and rms radius of an alpha particle for the model groundstate configurations. The dotted and dashed lines show how one can determine the parameters $\lambda$ and $e_{00}$ for a given compressibility $K_{0}$ and saturation density $\rho_{0}$. In the illustrated example the parameters $\rho_{0}=0.185 \mathrm{fm}^{-3}$ and $K_{0}=270 \mathrm{MeV}$ determine the values of $\lambda=0.4$ and $e_{00}=-16.79 \mathrm{MeV}$. These values of the parameters have been suggested by additional studies of the competition among Coulomb, surface and volume energies in the binding energy calculated for the ground-state configurations of the heavier nuclei (see table 1). This problem will be considered later on.

\subsection{Constraints on $e_{10}, L_{1}, K_{1}, e_{i i 0}, L_{i i}$ and $K_{i i}$ from the properties of $t$ and ${ }^{3} \mathrm{He}$}

After fixing the parameters $\rho_{0}, e_{00}, K_{0}$ and $\lambda$ let us try to examine the other ones. For $t$ and ${ }^{3} \mathrm{He}$ the ground-state energy does not depend on the choice of parameters $e_{i j 0}$,

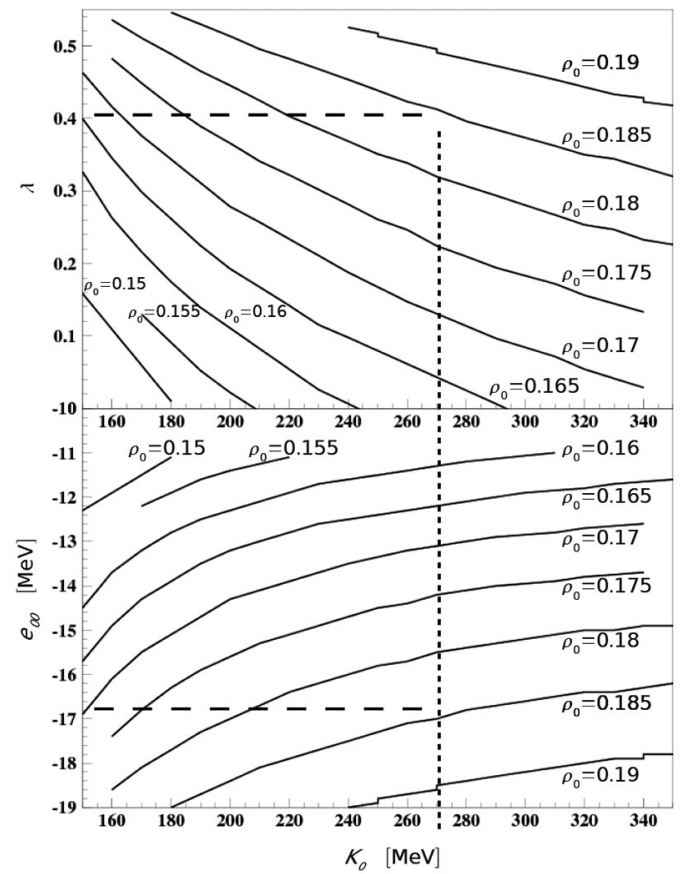

Fig. 2. Constant saturation density curves, $\rho_{0}$, as a function of $e_{00}, K_{0}$ and $\lambda$, constrained by the ground-state properties of an $\alpha$-particle.

Table 1. Parameters of the standard EoS which, together with a parameter of the surface energy $\lambda$, are reproducing the ground-state energy and rms radius of the alpha particle, $t$ and ${ }^{3} \mathrm{He}$.

\begin{tabular}{|c|c|c|c|c|c|c|}
\hline$\rho_{0}$ & $e_{00}$ & $K_{0}$ & $e_{I 0}$ & $L_{I}$ & $K_{I}$ & $\lambda$ \\
\hline \hline$\left(\frac{1}{\mathrm{fm}^{3}}\right)$ & $(\mathrm{MeV})$ & $(\mathrm{MeV})$ & $(\mathrm{MeV})$ & $(\mathrm{MeV})$ & $(\mathrm{MeV})$ & 1 \\
\hline 0.185 & -16.79 & 270 & 32 & 59.405 & -1250 & 0.4 \\
\hline
\end{tabular}

$L_{i j}$ and $K_{i j}$. This results from the vanishing of the $\eta_{n} \eta_{p}$ product in the last term of (6). Thus, the binding energies and rms radii for $t$ and ${ }^{3} \mathrm{He}$ can provide four equations for six unknown EoS parameters: $e_{I 0}, L_{I}, K_{I}, e_{i i 0}, L_{i i}$ and $K_{i i}$, and so, two equations are still missing. The two free parameters can be constrained from the properties of heavier nuclei as was done in the case of the fully symmetric nuclear matter. At this stage it is only possible to examine the subspace defining the limits for the considered parameters from the properties of the $t$ and ${ }^{3} \mathrm{He}$ nuclei. We notice the fact that the parameters associated with isospin, $e_{I 0}, L_{I}$ and $K_{I}$, differentiate between the binding energy and the rms radii of $t$ and ${ }^{3} \mathrm{He}$, while the parameters $e_{i i 0}, L_{i i}$ and $K_{i i}$ affect equally the energy and the size of these particles.

Using a similar method as in the case of an alpha particle one can find relationships among the parameters $e_{I 0}$, $K_{I}$ and $L_{I}$, by requiring a precise description of the binding energies and radii of the $t$ and ${ }^{3} \mathrm{He}$ particles. Here, the values of $e_{I 0}$ and $K_{I}$ were varied independently on a grid and the value of $L_{I}$ was found from the minimization of (17). The results of such calculations are shown in fig. 3 . 


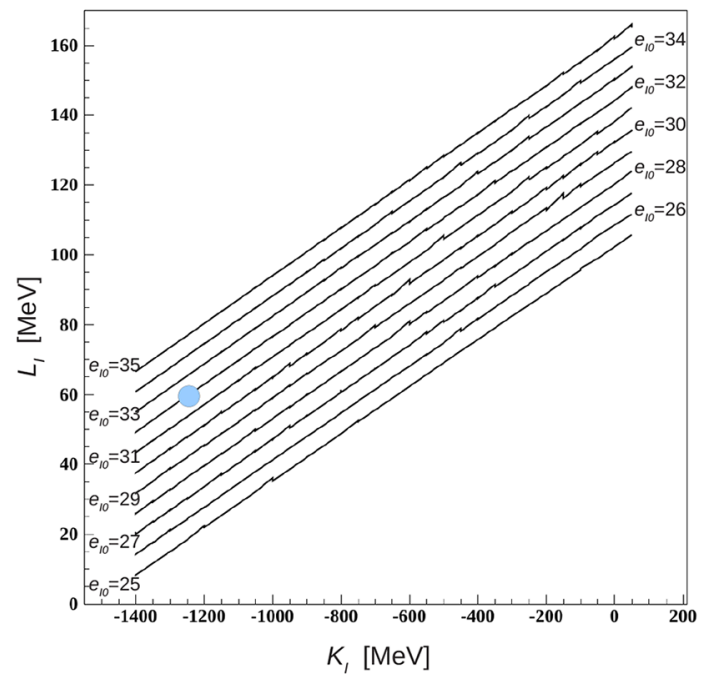

Fig. 3. Correlation between the slope, $L_{I}$, and curvature, $K_{I}$, of the isospin symmetry energy for different Wigner energies, $e_{I 0}$, obtained from fitting the binding energies and rms radii of the $t$ and ${ }^{3} \mathrm{He}$.

Table 2. Parameters of the spin interaction chosen in such a way that together with parameters from table 1 they reproduce binding energies and sizes of $d, t$ and ${ }^{3} \mathrm{He}$.

\begin{tabular}{|c|c|c|c|c|c|}
\hline$e_{i i 0}$ & $L_{i i}$ & $K_{i i}$ & $e_{i j 0}$ & $L_{i j}$ & $K_{i j}$ \\
\hline \hline$(\mathrm{MeV})$ & $\left(\frac{1}{\mathrm{fm}^{3}}\right)$ & $(\mathrm{MeV})$ & $(\mathrm{MeV})$ & $(\mathrm{MeV})$ & $(\mathrm{MeV})$ \\
\hline 82.37 & 242.48 & 19.909 & -1.5 & 182.0 & 1210.0 \\
\hline
\end{tabular}

The figure presents a relationship between the parameters $e_{I 0}, L_{I}$ and $K_{I}$. The selection of two of them allows to determine the third one. It should be stressed that the above correlations have been obtained as a result of the procedure reproducing the $t$ and ${ }^{3} \mathrm{He}$ ground-state properties with the parameters $\rho_{0}=0.185 \mathrm{fm}^{-3}, K_{0}=270 \mathrm{MeV}$, $\lambda=0.4$ and $e_{00}=-16.79 \mathrm{MeV}$ fixed from the groundstate properties of an alpha particle (fig. 2). Three other parameters, $e_{i i 0}, L_{i i}$ and $K_{i i}$, were found to be almost independent of $e_{I 0}$ and $K_{I}$.

\subsection{Discussion of the parameter values}

The final values of the EoS parameters adopted in this work have been obtained by taking into account also the binding energy and size of a deuteron. The values have been summarized in tables 1 and 2. For this example set of values the model reproduces exactly the experimental values of the rms radii and of the binding energies of the $d, t,{ }^{3} \mathrm{He}$ and $\alpha$ particles (see also figs. 5 and 7 ).

From the point of view of the present work, which focuses on the nuclear systems with even and equal numbers of protons and neutrons (i.e. the spin-balanced matter), the parameters $e_{i i 0}, L_{i i}, K_{i i}$ and $e_{i j 0}, L_{i j}, K_{i j}$ from the expansion (6) are irrelevant. However, they do play an important role when constructing the ground-state configurations and determining the properties of nuclei composed of asymmetric matter.
As far as the interactions between neutrons and protons are concerned, the Coulomb repulsion between protons causes that the proton matter tends to locate itself in the outer regions of the nucleus. This leads to differences between the neutron and proton density distributions, even for symmetric nuclei, and triggers additional interactions associated with the isospin symmetry energy (proton or neutron skins can be created). This effect can be negligible in the case of light nuclei, while for heavier ones it may be more significant. Therefore, for heavier nuclei, one has to use a reliable set of isospin symmetry energy parameters $e_{I 0}, L_{I}$ and $K_{I}$, even for symmetric nuclear matter.

As has been shown above, the binding energies and the rms radii of $t,{ }^{3} \mathrm{He}$ and $\alpha$ impose certain constraints on the parameters of the EoS. In the case of the $e_{00}, \rho_{0}$, $K_{0}$ and $\lambda$ parameters, once two of them are fixed by some other constraints or means, or from some other evidences, the remaining ones can be determined using the obtained correlations (fig. 2). Here, the compressibility parameter of symmetric matter $K_{0}$ should fall within the range of about $250 \pm 50 \mathrm{MeV}$ according to the recent experimental constraints (flow interpretation [20], monopole vibrations $[21,22]$, subthreshold kaon production [23, 24]). It has been fixed to $270 \mathrm{MeV}$ in the present approximation. The volume energy parameter $e_{00}$ should take the value of about $-16 \mathrm{MeV}$ according to the LDM. In the present approximation it has been assumed to have the value of $-16.79 \mathrm{MeV}$. The values of the remaining two parameters, $\rho_{0}$ and $\lambda$ have been then uniquely determined from the correlation plot of fig. 2 .

In the case of the symmetry energy, the choice of any two parameters out of $e_{I 0}, L_{I}, K_{I}$, allows to determine the third one (fig. 3). The compilation [25] of the latest results of the nuclear-physics experiments and astrophysical observations suggests that the value of the symmetry energy constant, $e_{I 0}$, and of the slope parameter, $L_{I}$, should fall within a range of about $32 \pm 1 \mathrm{MeV}$ and of about $59 \pm 17 \mathrm{MeV}$, respectively. In the present approximation the values of these parameters have been assumed to be 32 and $59.405 \mathrm{MeV}$, respectively. These values yield the value of $K_{I}$ to be about $-1250 \mathrm{MeV}$, according to the correlation plot of fig. 3 . The experimental constraint of [26] on the symmetry energy compressibility parameter, $K_{I}$, yields its value to be about $-50 \pm 200 \mathrm{MeV}$. Another compilation [19] locates the theoretical values of $K_{I}$ between -400 and $+466 \mathrm{MeV}$ and its experimental values in a range from $-566 \pm 1350 \mathrm{MeV}$ to $34 \pm 159 \mathrm{MeV}$. Thus, this parameter is least constrained experimentally and theoretically so far. Its value obtained within the model, preferring very soft symmetry energy, is consistent with the negative experimental constraint, thanks to its large error bar. However, we should stress that the adopted set of values is not at all unique. As seen from the correlation plots, some other choice of parameters is equally well possible. It should be also emphasized that the assumed value of $\rho_{0}$ equal to $0.185 \mathrm{fm}^{-3}$, which fits well the properties of the LCPs, and is higher than the commonly used values of $0.16-0.17 \mathrm{fm}^{-3}$, may influence the values of the remaining parameters. 


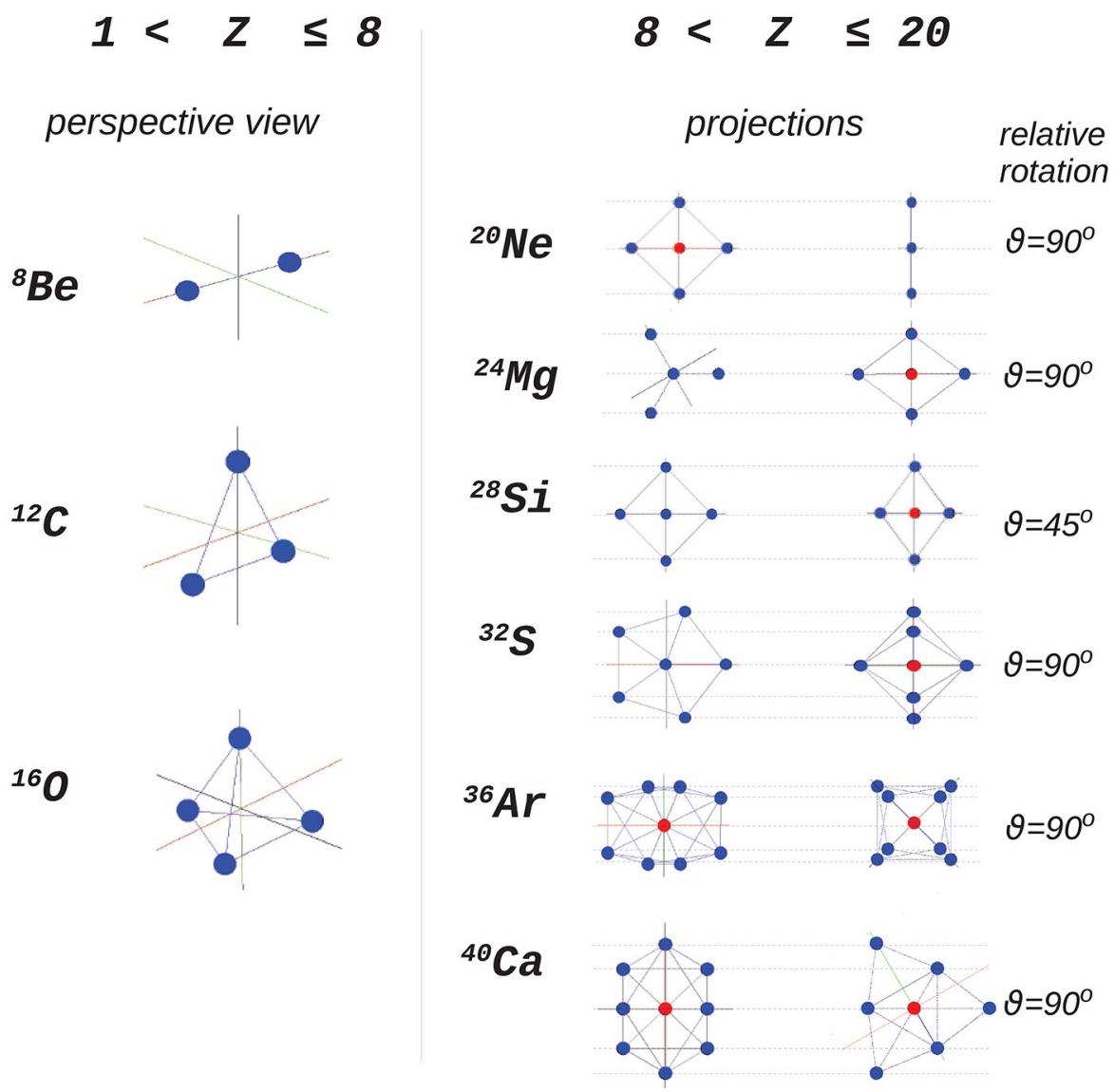

Fig. 4. Proposed structures of $n$-alpha nuclei. These structures ensure a high level of symmetry for a given number of alpha particles (see [3]). The density distribution of the central cluster (the red one) has quite a big spread and, therefore, it cannot be identified as an alpha particle.

In order to describe the exotic nuclei and provide more precise parameters of the symmetry energy, definitely some more advanced parameter searches will be needed, involving, e.g., fitting the properties of the neutron skin nuclei and also of those approaching the proton drip line across the chart of the nuclides, but this is beyond the scope of the present work.

A concise summary of the procedure of fixing the parameter values is presented in appendix $\mathrm{A}$.

\section{Energy and size of nuclei with equal and even number of neutrons and protons}

A method of finding the ground-state configuration based on minimization of the average value of the liquid-droplike Hamiltonian (16) with respect to the wave function parameters $\left\langle\mathbf{r}_{k}\right\rangle$ and $\sigma_{k}(r)$ with the $\left\langle\mathbf{p}_{k}\right\rangle=0$ constraint, is described in $[6,18]$. The ground-state configuration parameters determined in this way indicate that the corresponding wave function has some numbers of centers, which can be interpreted as the centers of alpha-like clusters in the nuclear matter. Some features of these ground-state configurations have been discussed in [6] without taking into account interactions and correlations among clusters. In the present approach the modification of the ground-state configurations due to the presence of the alpha-alpha interactions will be investigated.

The main aim is to identify such symmetries of the ground-state configuration, and thus of the corresponding wave function, which could be responsible for the experimentally observed changes of the binding energies and rms radii of the $n$-alpha nuclei as a function of $n$. We would like to check whether it is possible to identify specific configurations related to the magic numbers. In other words, we would like to verify whether the shell corrections necessary to describe deviations from the smooth predictions of the LDM around the magic numbers, could be interpreted in terms of a change of the ground-state configuration resulting from the corrections to the Hamiltonian related to the cluster-cluster interactions. As will be justified in the following, the ground-state configurations corresponding to the local minima of the Hamiltonian can be classified according to the nuclear charge, $Z$, in the following way:

i) $Z \leq 8$ : The structures are identical with the structures proposed in [2] and also in [6] and are characterized by no alpha cluster in the center of the system.

ii) $8<Z \leq 20$ : A core represented by one alpha-like cluster appears in the center of the system.

iii) $20<Z \leq 28$ : A core with a mass corresponding to two alpha particles appears in the center. 
The structures for cases i) and ii) are visualized in fig. 4 (see also [3]).

The structures presented in fig. 4 result from the analysis of a large number of configurations obtained within a procedure of minimizing the Hamiltonian (16) when starting from a random configuration (see the ${ }^{12} \mathrm{C}$ example in fig. 1). The majority of the thus-obtained configurations could be classified as those presented in the previous paper [6], with alpha-like clusters located mostly at the surface and with a moderate level of symmetry. Binding energies for these structures followed the smooth LDM trend and could not reproduce the "fine" structure of binding energies around the magic numbers. In some cases, however, the minimization procedure did not yield the global minimum, but ended up in some local one, just about $0.3-0.5 \mathrm{MeV}$ above the ground-state energy of majority of configurations. The configurations corresponding to these local minima were characterized by a higher level of symmetry and possibly contained alpha-like cluster(s) in the middle. Surprisingly, the binding energy distribution for these structures did show characteristic "kinks" around magic nuclei, despite some energy offset. These specific structures are those presented in fig. 4.

The question that arose from the above observation was whether it might be possible to convert the local minima into global ones, while keeping the highly symmetric structures, by introducing the corrections to the Hamiltonian which take into account the cluster-cluster interactions. The following sections try to verify this hypothesis.

\subsection{Matter without alpha clusters}

The structures from fig. 4 have been used as structural constraints in the minimization procedure by providing the starting points for the preformed alpha-like clusters. In other words, the random initial configurations, as in fig. 1, have been replaced by those from fig. 4 . The actual ground-state configurations were determined through minimization of the liquid-drop-like Hamiltonian (16) by varying the mean positions and the widths of the individual wave packets.

The following analysis is restricted to nuclei smaller or equal to ${ }^{40} \mathrm{Ca}$ which is the heaviest stable $n$-alpha nucleus. Beyond $Z=20$ the nuclei become radioactive and there are no data about their sizes available.

The liquid-drop-like Hamiltonian (16) with the parameters from tables 1 and 2 has been used together with structural constraints to obtain the ground-state configurations for each of the considered nuclei. The minimization procedure provided the final parameters determining the ground-state wave function: $\left\langle\mathbf{r}_{i}\right\rangle$ and $\sigma_{i}$. Figure 5 presents the obtained ground-state binding energies and rms radii as a function of the atomic number $Z$. The experimental data and the model results are represented by the squares and the circles, respectively. As one can see the calculated binding energies change their slope at the same place $(Z=8)$ as the experimental ones and the same concerns the radii. The overall calculated binding energies are somewhat bigger by about $0.3-0.5 \mathrm{MeV} /$ nucleon than the

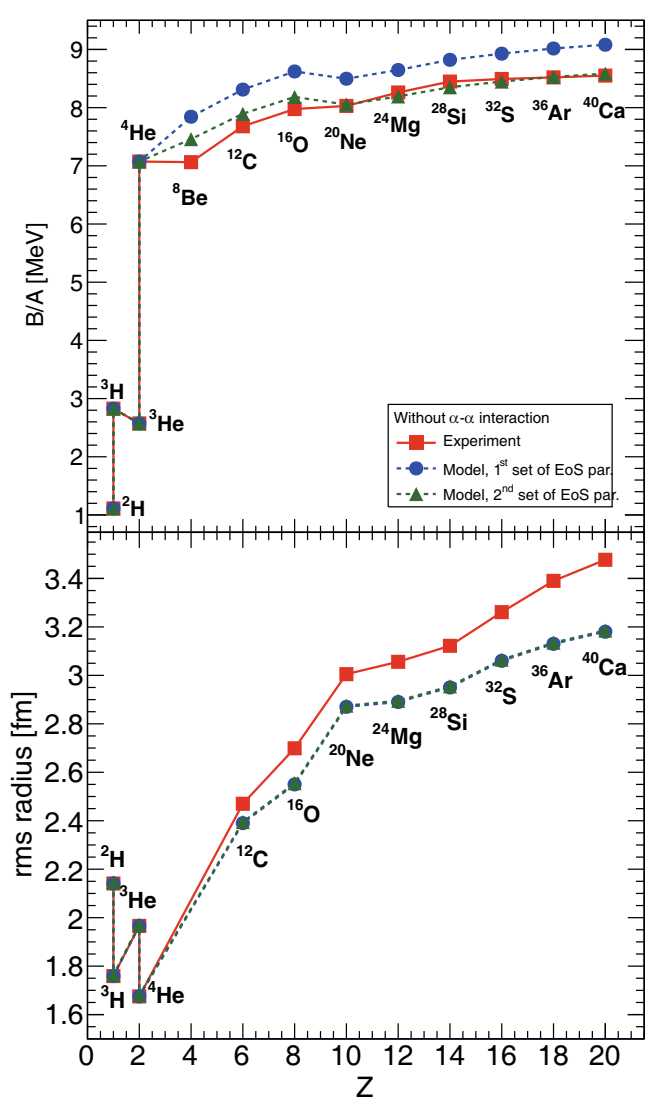

Fig. 5. The binding energies and rms radii of even-even nuclei $(N=Z)$ obtained for the ground-state wave functions and the Hamiltonian (16). The solid line connects the experimental points. Circles and triangles represent model results for two sets of parameters of the EoS.

experimental ones and the calculated radii are somewhat smaller; however both follow the experimental trends. The properties of particles up to ${ }^{4} \mathrm{He}$ are reproduced perfectly well, because they served as the constraints in the parameter fitting procedure.

In order to check the sensitivity of the results on the volume energy parameter $e_{00}$, similar calculations for $e_{00}$ equal to $-16.1 \mathrm{MeV}$ instead of $-16.79 \mathrm{MeV}$ have been performed. The results are presented with a dotted green line in fig. 5. In the case of the rms radii there is almost no difference observed. The binding energies still deviate from the experimental values, but what is important, similar changes of the slopes in theoretical and experimental trends are observed. Results of other trial calculations in which all the remaining parameters from $e_{0}$ to $\lambda$ were varied, did not yield a better reproduction of the experimental values with the use of the liquid drop form of the Hamiltonian together with the structural constraints i)-iii). A better reproduction could only be obtained with the corrections to the Hamiltonian discussed in the next section. The structural constraints assure configurations for which global minima of energy are obtained, provided that the alpha-alpha interactions are taken into account. Otherwise, they correspond to some local minima. 


\subsection{Modification of the Hamiltonian due to alpha clusters}

Let us consider now the formation of the nucleus by randomly located nucleons remaining within the range of their mutual interaction. Due to the Hamiltonian (16), nucleons are forming groups in such a way that the energy of the system is minimized. At a certain density of the matter alpha-like clusters can be formed as a result of the spinisospin interactions of nucleon groups. As alpha clusters are formed, additional extra interactions connected with multi-body effects can appear having a strong influence on the final structure of the system. Figure 6 illustrates a ground state of such a system. One can see that inside a matter drop alpha clusters appear and interactions between them are changing the energy of the system. Below, corrections to the Hamiltonian arising from these extra interactions will be defined.

Assuming that alpha-like clusters appear in the structure of the nucleus, one can expect two kinds of minor adjustments of the Hamiltonian, which should describe the essential part of the $\alpha$ - $\alpha$ interaction.

The first correction corresponds to the possible interaction caused by the appearance of specific many-body forces. Such many-body effect can be interpreted as a modification of the type and numbers of exchange bosons in the interaction process. It appears possible that, in such an exchange, the charge currents are strongly limited because they change the energy of the considered clusters. In this case, we can expect an additional term in the interaction potential which gives the repulsive forces between groups of nucleons forming a cluster of alpha-like particles (see fig. 4).

Here we test this hotfix in a form given by the product of the harmonic-oscillator potential $V_{\alpha \alpha}\left(d_{i j}\right)$ and probabilities $P_{\alpha}(i), P_{\alpha}(j)$ that, given four nucleons (groups named $i$ or $j$ ), form the alpha clusters:

$$
\left\langle\Phi\left|\Delta H_{1}\right| \Phi\right\rangle=\sum_{i \neq j} P_{\alpha}(i) P_{\alpha}(j) V_{\alpha \alpha}\left(d_{i j}\right),
$$

where the potential $V_{\alpha \alpha}\left(d_{i j}\right)$ is taken in the form

$$
V_{\alpha \alpha}\left(d_{i j}\right)=\kappa\left(d_{i j}-d_{0}\right)^{2}
$$

with $d_{i j}$ being the average distance between clusters $i$ and $j$, and $P_{\alpha}(i)$ describing the probability that a group of four nucleons $4 n(i)$ forms an alpha cluster $i$. We assume that $P_{\alpha}(i)$ can be expressed as

$$
P_{\alpha}(i)=\exp \left(-\nu \frac{\sigma_{4 n(i)}-\sigma_{\alpha 0}}{\sigma_{\alpha 0}}\right)^{2}
$$

where $\sigma_{4 n(i)}$ is the variance of the distribution describing the probability of finding a nucleon in a group $4 n(i)$ and $\sigma_{\alpha 0}$ describes such a variance (experimentally measured) for alpha particles. In the above formulas $\kappa, d_{0}$ and $\nu$ are free parameters. It is assumed that the potential is equal to 0 when $d_{i j}>4 \mathrm{fm}$ with a shape close to the van der Waals one.

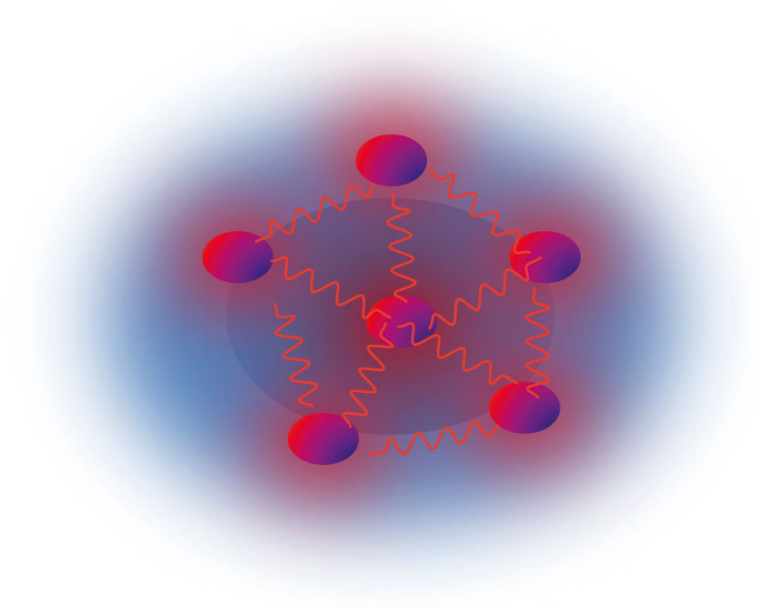

Fig. 6. Visualization of the additional interactions between alpha clusters due to the many-body interactions.

The second correction is related to the additional symmetry of the wave function, which can be associated with the bosonic character of an alpha cluster.

In the present approach nucleons and their clusters are distinguishable objects (1). For nucleons their energy connected with the fermionic motion is taken into account by applying the energy density functional. For alpha particles a relevant correction to energy should also appear and it is an empirical one.

When calculating the minimum of the Hamiltonian by varying the parameters of the wave function, the position of the alpha-like cluster and its size and spread are established.

These quantities can be different for different clusters depending on the adopted structural constraint for a given nucleus. The exchange of clusters must cause an increase of the energy of the system as a minimum of the Hamiltonian defines definite spreads of clusters for their different positions.

For the indistinguishable alpha particles due to the symmetrization with respect to the exchange of cluster positions there is no chance to relate cluster sizes to their positions.

The second correction is an empirical one:

$$
\left\langle\Phi\left|\Delta H_{2}\right| \Phi\right\rangle=\mu N_{\alpha} \sum_{i=1}^{i=A}\left(\frac{\sigma_{i}(r)-\sigma_{0}(r)}{\sigma_{0}(r)}\right)^{2}
$$

where $N_{\alpha}$ is the number of alpha clusters and $\mu$ is a parameter. $\sigma_{i}(r)$ is the variance of the nucleon radius and $\sigma_{0}(r)$ is the average variance of nucleons forming an alpha particle.

Thus, this correction to the Hamiltonian describes an increase of the energy caused by different sizes of clusters and it should vanish when the wave functions for all the clusters correspond to alpha particles in their ground states.

It seems that this correction could be related to the bosonic character of alpha particles. Both corrections are 
Table 3. Estimated parameters of corrections (18), and (21) to the Hamiltonian (16) coming from interactions between alpha clusters.

\begin{tabular}{|c|c|c|c|}
\hline$\mu$ & $\nu$ & $d_{0}$ & $\kappa$ \\
\hline \hline$(\mathrm{MeV})$ & {$[1]$} & $(\mathrm{fm})$ & $\left(\mathrm{MeV} / \mathrm{fm}^{2}\right)$ \\
\hline 1.0 & 9.0 & 3.77 & 5.2 \\
\hline
\end{tabular}

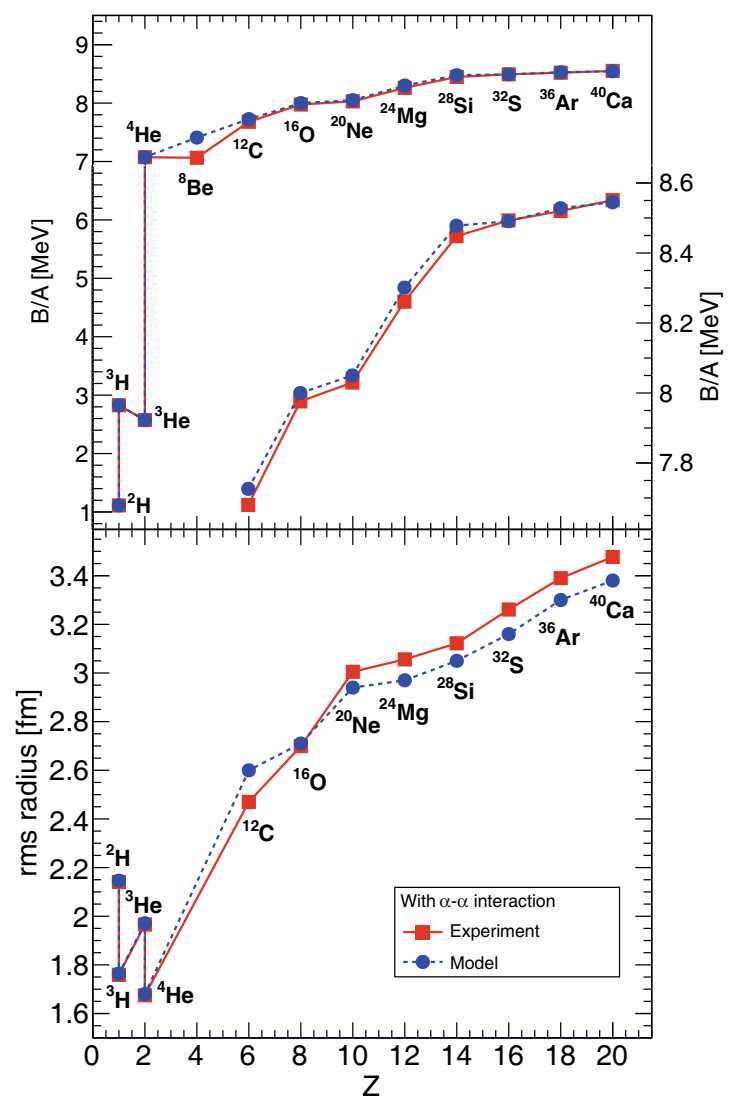

Fig. 7. Binding energies per nucleon and rms radii for the Hamiltonian with the corrections (18) and (21). The right scale of the binding energies per nucleon in the top panel refers to the magnified view for nuclei from ${ }^{12} \mathrm{C}$ to ${ }^{40} \mathrm{Ca}$. The solid (dashed) line connects the experimental (model) points.

positive and in this way the binding energy decreases. Assuming that parameters of the main part of the Hamiltonian (14) are established by properties of light nuclei $d, t$, ${ }^{3} \mathrm{He}$ and $\alpha$, the idea now is to define four additional parameters in order to reproduce the binding energies and sizes of nuclei starting from ${ }^{8} \mathrm{Be}$ up to ${ }^{40} \mathrm{Ca}$ by imposing the structural constraints.

The adopted values of these parameters are summarized in table 3 .

The results for the full Hamiltonian, including the corrections due to the alpha-alpha interactions are presented in fig. 7. The upper part of the figure shows the binding energies as a function of the atomic number $Z$. The bottom part presents the rms radii.

Much better agreement between the model results and the experiment can be observed now, as compared to fig. 5 .

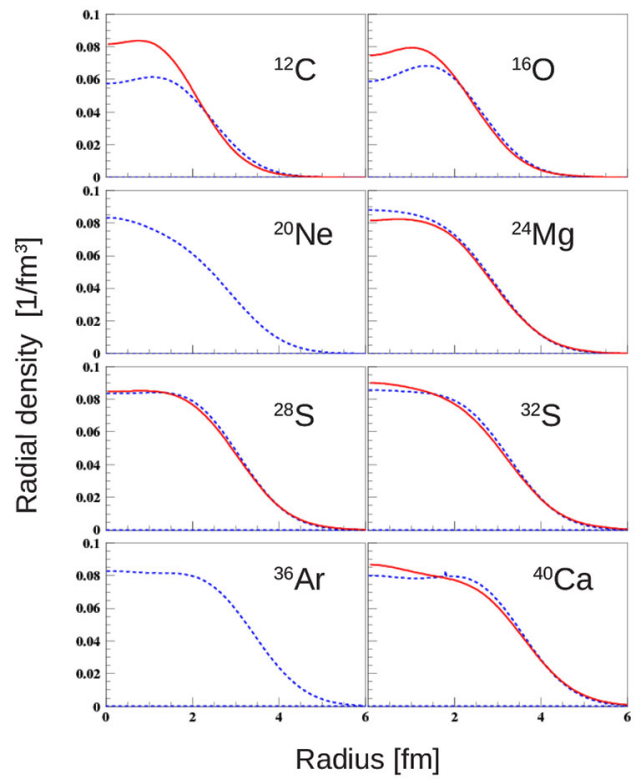

Fig. 8. Experimental (solid, red line) and model (dashed, blue line) density profiles.

Some discrepancies can be still noticed especially for ${ }^{8} \mathrm{Be}$ for which the distance between the alpha particles is pretty small and much smaller than $d_{0}$ in the harmonic potential description. Again, the properties of particles up to ${ }^{4} \mathrm{He}$ are reproduced perfectly well, because they served as the constraints to fix the EoS parameters.

Figure 8 presents a comparison of the model and experimental density profiles for the selected nuclei. The full (red) lines represent the experimental profiles and the broken (blue) lines result from the model calculations. Experimental density profiles were taken from [27]; however for ${ }^{20} \mathrm{Ne}$ and ${ }^{36} \mathrm{Ar}$ reliable experimental data were not available.

Figure 9 shows the isodensity contour plots for the model ground-state configurations of ${ }^{8} \mathrm{Be},{ }^{12} \mathrm{C},{ }^{16} \mathrm{O}$ and ${ }^{20} \mathrm{Ne}$. Note that apart from the ${ }^{16} \mathrm{O}$, which has a tetrahedral shape, all the other nuclei have a planar geometry (cf. fig. 4). A similar shape for ${ }^{12} \mathrm{C}$ has been obtained within the FMD [28] and AMD [29] simulations. In particular, the ${ }^{20} \mathrm{Ne}$ case shows that the central $\alpha$-like cluster is more diffused than the surface ones. More quantitatively, the standard deviations of the density distributions of surface alphas are of the order of $1.02 \mathrm{fm}$, while for the core alpha they are of the order of $1.28 \mathrm{fm}$.

Figure 10 presents a distribution of distances between alpha clusters for the nuclei in question.

The distances between clusters from the ground-state configurations obtained with the liquid-drop-like Hamiltonian without corrections are represented by open circles. The distances obtained from the full Hamiltonian calculations are represented by closed ones. As can be seen, in the latter case the distances are grouping around $3.5 \mathrm{fm}$. This can denote that these clusters behave more like rigid spheres. 


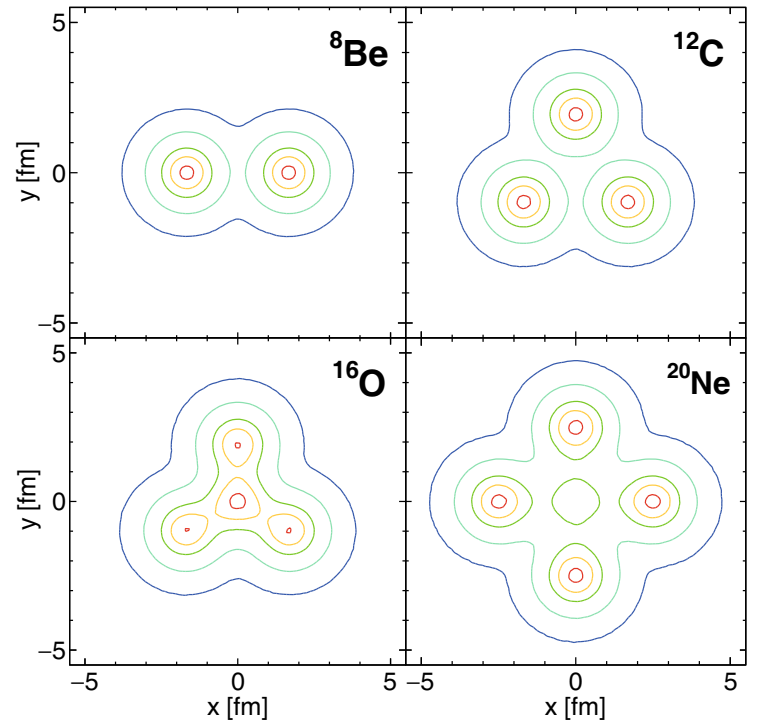

Fig. 9. Shapes of the model nuclei. The isodensity lines correspond to $1,15,50,75$ and $95 \%$ of the maximum density.

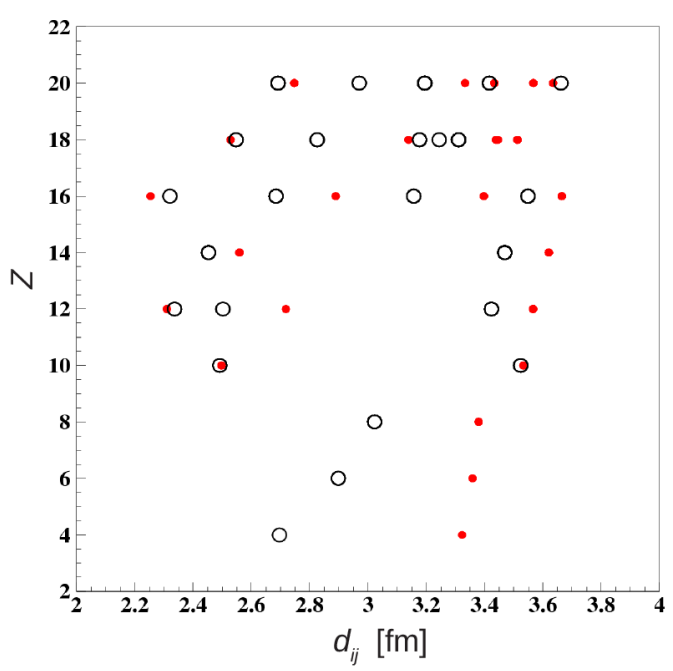

Fig. 10. The distribution of the distances between alpha clusters as a function of $Z$. The open (closed) circles represent the distances without (with) the correction for the alpha-alpha interaction in the Hamiltonian.

\section{Summary and conclusions}

A simple model of nuclei formed out of alpha-like clusters in which interactions lead to strong correlations between nucleons has been presented. The model interactions have been defined using a cubic approximation of the nuclear EoS. The proposed EoS contains additional terms which take into account the spin and isospin polarizations. These additional terms cause that nucleonic clusters, with an enlarged binding energy, appear in the ground-state configurations. These clusters have full spin-isospin symmetry similarly as in [8] and in [7]. For $n$-alpha nuclei these clusters can be identified as alpha-like structures. When these clusters are formed in regions of smaller density, i.e. at the nuclear surface, then their sizes measured as the vari- ance of the density distribution become comparable to the alpha-particle size. On the other hand, the formation of the clusters in the central part of nuclei leads to much bigger spreads of their density distributions and they can hardly be identified as alpha particles.

It has been shown that by taking into account the liquid-drop-like form of the Hamiltonian in the minimization process it was not possible to describe the subtle changes of the binding energies of nuclei. Therefore, the liquid drop part of the Hamiltonian has been supplemented by the corrections taking into account the clustercluster interactions. The ground-state configurations obtained from the minimization of the full Hamiltonian with the imposed structural constraints were found to reproduce very well the experimental binding energies and sizes of the $n$-alpha nuclei and the available density profiles. In particular, the model with the cluster corrections has been able to reproduce the "fine structure" of the ground-state energy distribution (inset of fig. 7). In this respect it seems to be able to mimic the shell effects without explicit spinorbit term.

The interactions have been defined using a 12-parameter EoS approximation. The thirteenth parameter has been added to control the variance of the field for finite systems. The values of these parameters and correlations among them have been constrained using the experimental binding energies and sizes of $d, t,{ }^{3} \mathrm{He}$ and $\alpha$ particles. Four additional parameters have been introduced in the corrections to the liquid drop part of the Hamiltonian in order to account for the interactions between the alpha-like clusters and to substantially improve the model predictions. The differences between the calculated and measured ground-state energies for nuclei with $Z>8$ are about an order of magnitude smaller than those obtained using the liquid drop model. Besides, the obtained parameters describe the properties of the $t,{ }^{3} \mathrm{He}$ and $\alpha$ very accurately (practically without error).

It has also been demonstrated that correlations between nucleons in the phase space can play an important role in the description of the ground states, particularly of the ground states of nuclei with the same and even number of neutrons and protons.

This work was supported by the Polish National Science Centre (NCN), contract Nos. UMO-2013/10/M/ST2/00624, UMO2013/09/B/ST2/04064, by IN2P3 08-128 and also by the Foundation for Polish Science MPD program. Stimulating discussions with Profs. Lucjan Jarczyk, Bogusław Kamys, Andrzej Magiera, Zbigniew Rudy, Roman Płaneta, Andrzej Wieloch and Janusz Brzychczyk are gratefully acknowledged. Special thanks to are due the anonymous referee for the many suggestions that improved the original version of the manuscript.

\section{Appendix A. Extraction of the EoS parameters}

The Hamiltonian (16) depends on 13 parameters and since the binding energies and rms radii of the ground states 


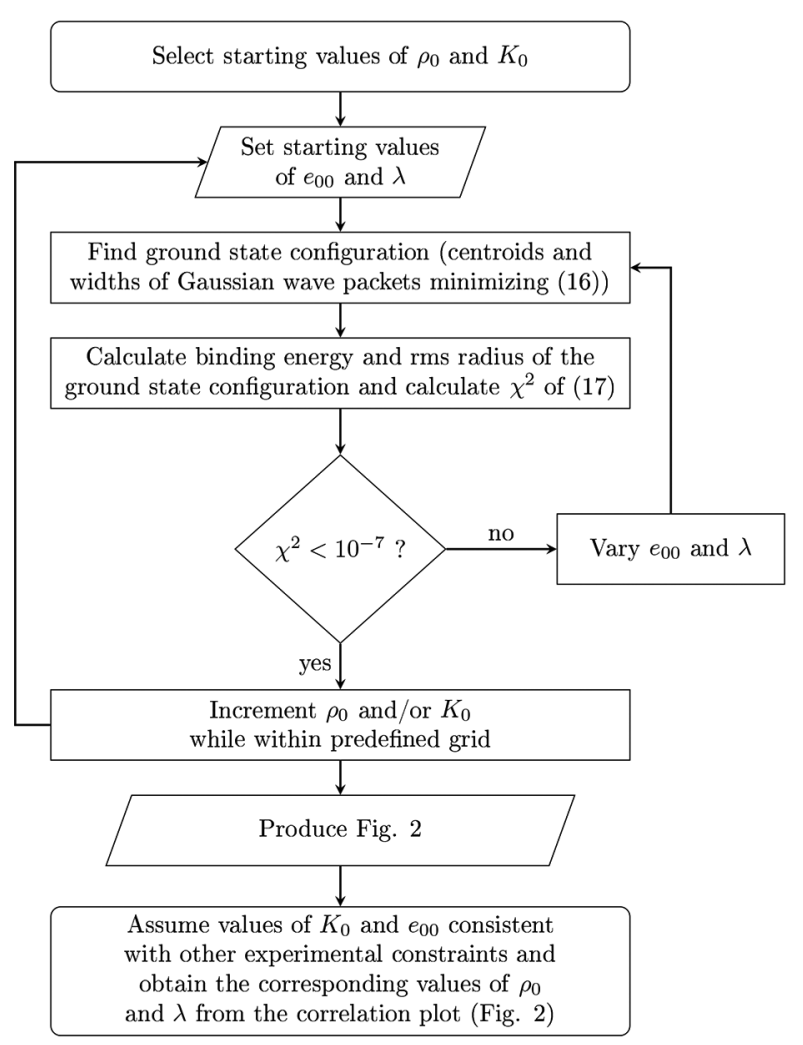

Fig. 11. Flow chart of the procedure for fixing the $K_{0}, \rho_{0}, e_{00}$ and $\lambda$ parameters using the binding energy and the rms radius of an alpha particle as a constraint. The details can be found in sect. 3 .

of $d, t,{ }^{3} \mathrm{He}$ and $\alpha$ provide only 8 constraints, 5 of the parameters have to be fixed by some other means.

The first step of the procedure to fix the EoS parameters consisted in constraining the values of $K_{0}, \rho_{0}, e_{00}$ and $\lambda$ by the binding energy and rms radius of an alpha particle. This step has been summarized in the flow chart of fig. 11.

The compressibility parameter of symmetric matter $K_{0}$ and the volume energy parameter $e_{00}$ have been fixed within the range of the recent experimental constraints. The values of the remaining two parameters, $\rho_{0}$ and $\lambda$, are then uniquely determined from the correlation plot of fig. 2. The remaining 9 parameters were irrelevant for the properties of an alpha particle, which greatly simplified the corresponding EoS.

In the second step, the parameters $e_{I 0}, L_{I}$ and $K_{I}$ have been found in an analogous way by using the binding energies and the rms radii of $t$ and ${ }^{3} \mathrm{He}$ as constraints in eq. (17), by fixing the previously determined parameters $K_{0}, \rho_{0}, e_{00}$ and $\lambda$, and by disregarding the remaining 6 parameters.

Finally, the remaining 6 parameters $e_{i i 0}, L_{i i}, K_{i i}, e_{i j 0}$, $L_{i j}$ and $K_{i j}$ have been found by minimizing (17) with the fixed 7 parameters determined in the previous two steps and using the binding energies and the rms radii of $d, t$ and ${ }^{3} \mathrm{He}$ as constraints. Fitting is done in an analogous way as in the inner loop of the flow chart of fig. 11, replacing the $e_{00}$ and $\lambda$ parameters by the remaining 6 ones.
After fixing the 13 parameters of the liquid-drop-like Hamiltonian (16), the additional four parameters of the $\alpha-\alpha$ interaction, $\mu, \nu, d_{0}$ and $\kappa$, have been fixed by fitting the ground-state binding energies and rms radii of the nuclei from ${ }^{8} \mathrm{Be}$ up to ${ }^{40} \mathrm{Ca}$. Here, in addition, the structural constraints i)-iii) have been imposed in the fitting routine, meaning that only the widths of the nucleon wave packets and the inter- $\alpha$-like clusters distances could be varied, preserving the imposed configuration.

The procedure of finding the ground-state configurations which minimize the model Hamiltonian is described in sect. 4 of [18], see also fig. 1.

Open Access This is an open access article distributed under the terms of the Creative Commons Attribution License (http://creativecommons.org/licenses/by/4.0), which permits unrestricted use, distribution, and reproduction in any medium, provided the original work is properly cited.

\section{References}

1. H.A. Bethe, R.F. Bacher, Rev. Mod. Phys. 8, 82 (1936).

2. L.R. Hafstad, E. Teller, Phys. Rev. 54, 681 (1938).

3. http://nz21-33.ifj.edu.pl/clusters/.

4. M. Freer, Scholarpedia 5, 9652 (2010) doi:10.4249/scholarpedia.9652.

5. W. von Oertzen, Eur. Phys. J. A 29, 133 (2006).

6. Z. Sosin, J. Kallunkathariyil, Acta Phys. Pol. B 45, 925 (2014).

7. H. Feldmeier, Nucl. Phys. A 515, 147 (1990).

8. A. Ono et al., Prog. Theor. Phys. 87, 1185 (1992).

9. Y. Kanada-En'yo et al., Prog. Theor. Phys. 2012, 01 A202 (2012).

10. L. Wilets et al., Nucl. Phys. A 282, 341 (1977).

11. C. Dorso, S. Duarte, J. Randrup, Phys. Lett. B 188, 287 (1987).

12. H. Horiuchi, A. Ohnishi, T. Maruyama, in Proceedings of the 6th International Conference on Nuclear Reaction Mechanisms, Varenna, June 10-15, 1991, edited by E. Gadioli, Ricerca Scientifica ed Educazione Permanente, Suppl. No. 84 (Univeristà degli Studi di Milano, 1991).

13. D.H. Boal, J.N. Glosli, Phys. Rev. C 38, 1870 (1988).

14. T. Maruyama et al., Phys. Rev. C 53, 297 (1996).

15. J. Fink et al., Z. Phys. A 323, 189 (1986).

16. J. Aichelin, Phys. Rep. 202, 233 (1991).

17. P. Dirac, The Principles of Quantum Mechanics, Oxford Science Publications Series (Oxford University Press, 1981).

18. Z. Sosin, Int. J. Mod. Phys. E 19, 759 (2010).

19. Bao-An Li et al., Int. J. Mod. Phys. E 07, 147 (1998).

20. P. Danielewicz et al., Science 298, 1592 (2002).

21. J. Piekarewicz, Phys. Rev. C 69, 041301 (2004).

22. G. Colò et al., Phys. Rev. C 70, 024307 (2004).

23. C. Sturm et al., Phys. Rev. Lett. 86, 39 (2001).

24. Ch. Hartnack et al., Phys. Rev. Lett. 96, 012302 (2006).

25. Bao-An Li, Xiao Han, Phys. Lett. B 727, 276 (2013).

26. M. Centelles et al., Phys. Rev. Lett. 102, 122502 (2009).

27. H. De Vries, C.W. De Jager, C. De Vries, At. Data Nucl. Data Tables 36, 495 (1987).

28. H. Feldmeier, J. Schnack, Prog. Part. Nucl. Phys. 39, 393 (1997).

29. Y. Kanada-En'yo, H. Horiuchi, Prog. Theor. Phys. Suppl. 142, 205 (2001). 